\title{
L'emprise de l'occulte sur la légitimité de l'état et l'aide à la démocratisation en Afrique
}

\author{
Dirk Kohnert ${ }^{1}$
}

Caricature : «ne nous sommes-nous pas rencontrés à Harvard? » ${ }^{2}$

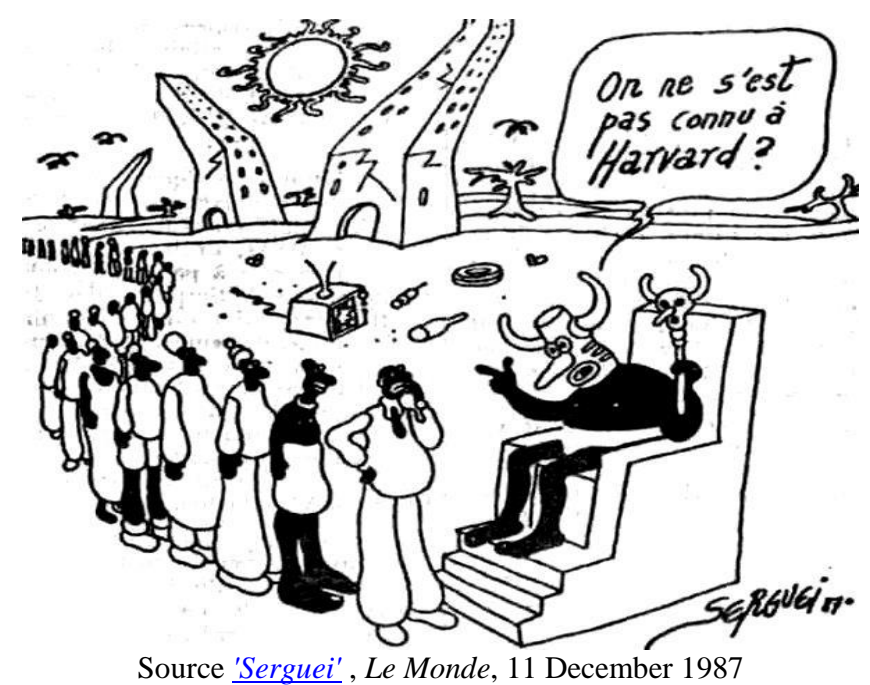

Résumé: Parmi les politiciens et les experts du développement en Afrique de même il y a une prise de conscience croissante de l'importance jamais décroissante de la croyance en la magie et la sorcellerie sur la prise de décision politique depuis l'époque précoloniale. La démonstration du contrôle des forces occultes comme un moyen de renforcer leurs légitimité avait été pendant plus d'un siècle la prérogative des chefs traditionnels et leurs marabouts. Aujourd'hui, il est efficace pour l'élite politique moderne et l'état moderne ainsi. Un nombre croissant d'états africains ont officiellement reconnu l'existence de la sorcellerie et de la magie, et ils adaptent la loi coloniale imposée en conséquence. En addition des systèmes de croyances magicoreligieuses, représentée par le vodun ou des églises indépendantes africaines (par exemple, les Kimbanguistes), bénéficiant de contrôler la sorcellerie, sont promus par les dirigeants politiques africains pour renforcer la légitimité à la fois de la classe politique et de la gouvernance de l'État. Dans le même temps, des experts en développement ont essayé de prendre en compte la dimension culturelle du développement socio-culturelle. Ils ont appelé à une «endogénéisation» de l'aide au développement. Cet appel a été justifié, parce que «l'endogénisation» devrait être considérée comme un prérequis de l'aide durable. Cependant, sous certaines conditions, il peut être ambigu et dangereux aussi. En ce qui concerne la considération de la croyance occulte elle peut conduire à la promotion de l'État illégitime et à la violation des droits humains fondamentaux.

Mots-clés: religion, sorcellerie, croyance occulte, théorie conspirationniste, développement durable, démocratisation, légitimité, aide au développement, Afrique subsaharienne, études africaines, Togo, Nigéria, Zaïre

JEL-code: F35, N37, N97, Q17, O29, O35, P48, Z12, Z13

\footnotetext{
${ }^{1}$ L'Institut des Affaires Africaines, GIGA-Hambourg, 1'Allemagne. Version révisée et améliorée de l'auteur en français de: (1997): Zum Einfluß des Okkulten auf staatliche Legitimität und Demokratisierungshilfe in Afrika, Sociologus, 47.1997.1: 24-50. Layout revised, hyperlinks to sources added, 13 janvier 2022.

2: Caricature sur la modernité des croyances occultes dans l'Afrique contemporaine, indépendamment du sexe, de la religion, de l'affiliation politique et de la classe sociale. --- Source 'Serguei', dessinateur français, Serguei Goizauskas, En: Jacques de Barrin, Le retour des sorciers - Désenchantement et traditions en Afrique. Le Monde, 11 December 1987, p. 44.
} 


\section{L'influence des systèmes de croyances occultes sur la politique et le pouvoir en Afrique est omniprésente}

En Afrique, la croyance en des personnes qui utilisent des pouvoirs surnaturels à leurs fins et exercent une magie noire n'est pas un phénomène marginal ou un trait socioculturel exotique qui est bouleversé par des ethnologues qui sont déconnectés de la réalité. De même, la croyance aux sorcières n'est pas une relique disparue de l'époque des civilisations de l'Afrique précoloniale, dont la pertinence est désormais limitée à quelques groupes ou régions ethniques dans les coins les plus reculés. Largement indépendante de l'orientation religieuse ou du niveau d'éducation, cette croyance - dans toutes les classes sociales - est toujours un déterminant essentiel de la pensée et de l'action de nombreuses personnes, en particulier en Afrique de l'Ouest ${ }^{3}$. Pour de nombreux Africains, la sorcellerie représente une véritable menace existentielle - selon les circonstances, parfois latente, parfois immédiate. À leurs yeux, cette menace est moins une question de forces surnaturelles diffuses et impersonnelles que, plutôt, très concrètes et directement, du mal potentiellement omniprésent chez nos semblables eux-mêmes ${ }^{4}$ (voir Douglas 1970).

Contrairement à la croyance populaire des Occidentaux, rien n'indique jusqu'à présent que la croyance en la magie et la sorcellerie s'affaiblit au cours du «processus de modernisation » en Afrique. Loin de là, aux yeux de nombreux Africains, la sorcellerie a plutôt augmenté en fréquence et en virulence au cours des dernières décennies. Cela est attribué, entre autres, à une réponse psychosociale à la «crise africaine» en général, et au potentiel de conflit croissant du processus en cours de différenciation sociale en Afrique en raison de la « modernisation » de l'économie et de la société.

Comme la plupart des religions, les systèmes de croyances occultes tentent d'établir un lien entre le connu et l'inconnu. Par une réduction de la complexité, ils tentent de trouver un ordre

\footnotetext{
${ }^{3}$ Un aperçu de l'état d'avancement de la discussion internationale sur le sujet a déjà été fourni ailleurs (voir Kohnert 1996). Seules deux sources africaines sont citées ici: Selon le théologien nigérian J. Omosade Awolalu: "aucune croyance (n'est) plus profondément enracinée que celle de l'existence des sorcières ... Pour les Yorùbá ainsi que pour d'autres groupes ethniques en Afrique, la sorcellerie est une réalité. C'est une croyance très répandue parmi les lettrés et les analphabètes, parmi les hauts et les bas de la société. "(Awolalu 1979: 81, traduction de l'anglais, D.K.). - Une commission interdisciplinaire du gouvernement de la province du Nord de l'Afrique du Sud (Limpopo), composée d'ethnologues, de théologiens et de juristes, d'avocats et de fonctionnaires, chargés d'enquêter sur la chasse aux sorcières dans les pays d'origine (1987-1994) est arrivée au même résultat: «De les déclarations ci-dessus, il devient très clair que personne ne peut maintenant affirmer que la sorcellerie est un mythe qui ne peut exister que dans l'esprit des ignorants »... "La fréquence continue des meurtres de sorcellerie, sinon leur augmentation, mérite une mention spéciale. De plus, la croyance en la sorcellerie reste aussi répandue que jamais. La croyance en la sorcellerie et les pratiques connexes font partie d'un principe culturel, traditionnel et coutumier fondamental des Africains en Afrique du Sud et en Afrique dans son ensemble. » (Ralushai et al 1996: 56-57 ; traduction de l'Anglais, D.K. ).

${ }^{4}$ La distinction classique de Evans-Pritchards (1937: 8-9) entre la sorcellerie inhérente, souvent héritée, ou le mauvais pouvoir psychique d'une personne (appelée «sorcellerie ») d'une part, et la magie noire délibérément utilisée, c'est-à-dire l'utilisation volontaire des connaissances locales d'experts en sciences naturelles en rapport avec les rites magiques afin de nuire aux autres (magie noire), ne semble pas être utile dans ce contexte de problèmes de chasse aux sorcières dans les 'homelands' (Bantoustan) de l'Afrique du Sud. Cela est d'autant plus vrai que la distinction d'Evans-Pritchard ne s'applique apparemment même pas dans d'autres sociétés africaines, en dehors de l'Azande (Zandé) (voir aussi Turner 1964; Macfarlane 1970a; Niehaus 1995: 513; Fisiy / Geschiere 1990: 136). - Ici et dans ce qui suit, la croyance en la sorcellerie se réfère à une conviction, que certaines personnes utilisent des forces occultes sombres par des motifs illégitimes - généralement égoïstes - pour nuire aux autres. Selon une conception émique, la sorcellerie est en principe «mauvaise », mesurée par les normes de légitimité en vigueur dans une société (voir Geschiere 1995: 267). Pour l'ambivalence du transfert des concepts péjoratifs euro-centristes de magie et de sorcellerie au contexte africain, voir cependant Geschiere (1995: 282) et la littérature citée.
} 
surnaturel dans le chaos apparent de l'univers. La croyance aux sorcières satisfait un besoin humain de confirmation de notre propre importance profondément enraciné, que le monde se préoccupe de nous-mêmes et de notre destin et que rien n'est laissé au hasard. La justification du malheur, de la maladie ou de la mort avec l'ensorcellement de personnes méchantes rend un monde supportable, qui autrement devrait être perçu comme profondément blessant, froid et impersonnel (voir Nadel 1954: 163-64, 205).

La croyance de la sorcellerie a des contenus et des caractéristiques différents, selon le mode de production dans lequel elle est ancrée et son cadre socio-culturel et socio-économique (voir Ardener 1970; Kohnert 1983; Nadel 1952). Par conséquent, nous devons mettre en garde contre les généralisations. Néanmoins, en plus des faits énumérés ci-dessus, il existe causes et effets communs établis de la croyance occulte en Afrique subsaharienne. Par exemple, la croyance en la sorcellerie elle-même (par opposition à la pratique de la persécution) n'est généralement pas liée aux groupes spécifiques d'âge et de sexe différents. Il est également pleinement compatible avec différents niveaux d'enseignement et différents religions, du moins en ce qui concerne la pratique quotidienne de la religion. De plus, depuis l'époque précoloniale, des dirigeants islamiques, comme des notables du nord des Émirats nigérians ont instrumentalisé la croyance occulte, c'est-à-dire qu'ils ont utilisé la croyance en la magie et la sorcellerie, tout aussi bien que les dirigeants des églises chrétiennes, comme moyen de subjuguer le peuple. Plus récemment, des églises fondamentalistes africaines pentecôtistes, proliférant dans toute l'Afrique, qui ont intégré les idées soi-disant "primitives païennes », diabolisées par les missionnaires européens, dans leur conception de l'univers (par exemple les pentecôtistes, la Rose-Croix et l'Église du christianisme céleste (ECC) en Afrique de l'Ouest), exercent aujourd'hui une attraction considérable ${ }^{5}$. Même de hauts dignitaires chrétiens, comme l'évêque de Kinshasa, croient à la sorcellerie, une croyance qui, selon eux, est partagée par $80 \%$ des Africains.

La croyance aux forces occultes a un impact profond sur l'éthique ainsi que sur la pensée et l'action des Africains. Par exemple, il joue un rôle crucial dans l'engagement des groupes sociaux en conflit en Afrique subsaharienne. Ses effets ne se limitent pas au secteur privé ou à la société civile, mais la croyance affecte également la classe politique et l'appareil d'État. Les systèmes de croyance occulte sont un déterminant essentiel du processus de prise de décision des politiciens et des fonctionnaires africains à tous les niveaux, du chef de l'État et de son gouvernement jusqu'au maire du village ou au l'agent de vulgarisation agricole d'État. Il est évident que cet effet a des implications importantes pour le processus de construction de l'État en général et le processus actuel de démocratisation en Afrique en particulier.

Depuis la fin des années 1980, les spécialistes en sciences sociales ont de plus en plus problématisé la dimension politique des systèmes de croyances occultes au niveau de l'État (voir Geschiere 1996: 83; 1995; Fisiy / Geschiere 1990; Comaroff 1993; Nadel 1954; Niehaus 1993; Schatzberg 1993). Néanmoins, les experts du développement et les politiciens des pays donateurs qui conseillent les gouvernements africains sur la mise en œuvre du processus de démocratisation, à quelques exceptions près, n'ont jusqu'à présent même pas pris note du problème de l'entrelacement des systèmes de croyances occultes, du pouvoir et de la légitimité des États. L'intégration tant annoncée de la «dimension socioculturelle » de la coopération au développement dans les conseils politiques des donateurs en Afrique reste cependant superficielle lorsque cet aspect essentiel de la culture africaine est rejeté comme une «marginalité exotique » sans histoire.

\footnotetext{
${ }^{5}$ Voir par exemple. Meyer, B. 1992 sur la lutte des groupes pentecôtistes contre les adzetwo (sorcières) dans le sud-est du Ghana.
} 
L'article suivant se concentre sur la clarification du lien général entre le pouvoir légitime et le pouvoir occulte ainsi que sur les questions de l'influence de la croyance de la sorcellerie sur la légitimité de l'État et son ancrage dans des systèmes juridiques socio-culturellement adaptés de l'État.

L'expérience sous-jacente pour les analyses suivantes est fournie par des recherches sur le terrain sur les effets sociaux, économiques et politiques de la sorcellerie dans le nord du

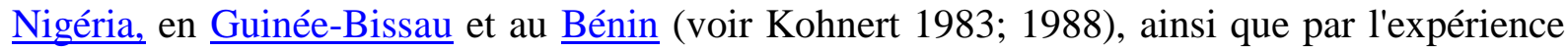
en tant qu'observateur électoral en Afrique. Des analyses secondaires comparatives d'études de cas publiées sur le sujet en Afrique subsaharienne et dans les Caraïbes (par exemple, le

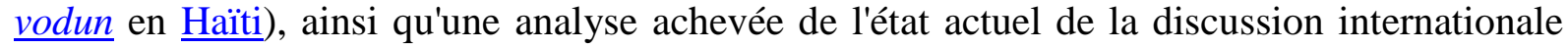
sur le sujet complètent l'étude (voir Kohnert 1996).

\section{La croyance en la sorcellerie est un pilier de différentes formes du pouvoir légitime}

Le processus de construction de l'État et de la démocratisation est inextricablement lié aux questions de légitimité de l'État et de ses dirigeants politiques. L'expérience antérieure avec l'observation des élections en Afrique depuis 1989, c'est-à-dire le début de la deuxième vague de la démocratisation, montre que des élections justes et libres, basées sur un système multipartite ouvert, peuvent apporter une contribution significative à l'amélioration de la légitimité de l'État (voir aussi Engel et al 1995 : 2-9,26 -29,178-86). La même expérience montre cependant que des élections fausses ou de façade sont utilisées par des régimes antidémocratiques et des autocrates pour aveugler leurs électeurs et prétendre avoir une (fausse) légitimité de la loi au pays et à l'étranger. Les élections présidentielles en Gambie (septembre 1996) ou les élections présidentielles et parlementaires sous le régime Eyadéma du Togo $(1993,1994 / 96)$ en sont des exemples notoires.

Aux yeux des personnes concernées, la véritable légitimité de l'État et de ses dirigeants politiques n'est pas créée, consolidée ou documentée uniquement ou principalement par la démocratisation formelle et les élections. Au moins dans le contexte africain, la légitimité politique a plusieurs sources. Dans les conditions politiques, économiques et sociales inadéquates d'un «État de commandement» africain [Kommandostaat, en allemand, voir Bierschenk, Elwert, Kohnert, 1993], et face à un système de favoritisme, à savoir le néopatrimonialisme fermement ancré dans de nombreux pays africains, la légitimité politique est une condition préalable nécessaire, mais nullement suffisante, à toute démocratisation durable à la base (voir Schatzberg 1993: 458; Geschiere 1996; Engel et al 1995: 1-28).

De l'avis de la majorité de la population concernée, cette légitimité n'est pas tant déterminée par les conditions du cadre politique formel (par exemple, la stabilité politique; le système multipartite; les élections) mais plutôt par l'emploi de structures socioculturelles traditionnelles et de visions du monde. Les deux ne sont en aucun cas pas statiques, mais sont sujets à des changements permanents (voir Geschiere 1995; Kohnert 1983). Une partie intégrante de ces structures qui créent la légitimité en Afrique subsaharienne sont les systèmes de croyance occulte, qui ont été, et sont toujours, les piliers essentiels de la légitimité des dirigeants politiques et religieux traditionnels tels que les émirs et les obas du Nigéria depuis la période précoloniale. 
Selon les croyances traditionnelles partagées par de nombreux Africains - probablement la plupart - aujourd'hui, le pouvoir occulte et «surnaturel » peut être aussi réel et direct que le pouvoir profane. La magie néfaste ou les méfaits des sorcières sont tout aussi sensibles pour de nombreux Africains, et rationnellement vérifiables, que la punition infligée par le pouvoir judiciaire de l'État. Mais même pour les spécialistes des sciences sociales occidentaux, les systèmes de croyances occultes sont tout sauf «irrationnels » (voir Evans-Pritchard 1937: 330; Gluckman 1944), bien que sa justification empirique ne soit guère compatible avec les catégories de la pensée scientifiquement façonnée. Mais la structure méthodologique de la sorcellerie-croyance est tout aussi rationnelle que celle des lignes directrices éthiques pour diriger nos actions, comme l'éthique de l'esprit protestant du capitalisme, elle ne fait que suivre une rationalité différente. Précisément, parce que le pouvoir occulte ne diffère pas de manière significative de la perspective émique des Africains concernés en termes de sa réalité et dans l'utilisation rationnellement prévisible du pouvoir du monde, chaque gouvernement en Afrique qui ne veut pas s'appuyer sur la force brute, doit faire ses preuves dans les deux sphères de pouvoir si elle veut être considérée comme efficace et légitime par ses citoyens.

Les accusations de sorcière et le pouvoir occulte sont généralement liés à certaines personnes. Pour cette raison, les dirigeants africains et leurs marabouts, par exemple, qui se vantent tous deux de posséder un pouvoir magique, doivent le prouver personnellement encore et encore, sinon ils se verront refuser la capacité de gouverner. Par conséquent, il serait seulement raisonnable de supposer que notamment ces types de pouvoir légitime sont soutenus par des systèmes de croyances occultes qui sont essentiellement liés à la personne, tels que le pouvoir charismatiques et traditionnelles au sens de la typologie de Max Weber (1972: 125 -30). La magie et la croyance aux sorcières seraient donc considérées comme leurs alliées naturelles. Cependant, partout en Afrique, nous trouvons des exemples, que l'attribution de la sorcellerie s'applique non seulement aux «dirigeants traditionnels » mais aussi à la nouvelle classe politique des dirigeants politiques modernes qui ont repris les affaires de l'État après l'indépendance. Très souvent, ces dirigeants ont été éduqués dans des écoles d'élite occidentales et des terrains de formation supérieurs tels que l'ENA français. Presque tous les chefs d'État africains - non seulement la vieille garde politique, comme Houphouët-Boigny (Côte d'Ivoire), Ali Bongo (Gabon), Eyadéma (Togo), Kérékou (Benin), Nkrumah (Ghana), Mobutu (Zaire) - mais aussi les nouveaux politico-technocrates, ne pouvaient s'empêcher d'utiliser ce recours aux systèmes de croyances occultes quand l'occasion s'est présentée. Un exemple notable est l'ancien président du Bénin, Nicéphore Soglo, qui a remplacé le dictateur Mathieu Kérékou lors de la première élection présidentielle multipartite du pays en 1991, annoncée la deuxième vague de la démocratisation en Afrique.

Pour les systèmes de planification calculés et orientés vers les objectifs, fondés sur la croyance en des règles et statuts établis, le recours à la croyance de la sorcellerie comme source de légitimité serait superflu. Cela s'appliquerait particulièrement à l'État constitutionnel moderne, tel que propagé par la communauté internationale des donateurs dans le cadre du conditionnement politique de l'aide au développement. Dans un tel État, les politiciens et les fonctionnaires devraient prendre des décisions indépendamment de la personne et de son statut social. La « domination rationnelle-légale » au sens de Max Weber (1972: 125-30), fondée sur la reconnaissance de l'attribution, bâtie sur des règles d'obligations d'exécution, de l'autorité et des moyens de coercition, plutôt que sur la reconnaissance des personnes, serait donc libérée du joug de la croyance al la sorcellerie.

Malheureusement, c'est une illusion. C'est une tromperie, non seulement parce qu'en réalité, la délimitation idéologique du pouvoir sous cette forme pure ne se produit jamais. Le pouvoir 
traditionnelle, charismatique et légale dans des pays africains (et d'ailleurs) d'aujourd'hui est plutôt inséparable.

En effet, dans le contexte africain, la « domination légale » doit tenir compte de la magie et de la sorcellerie si elle veut assurer sa légitimité aux yeux de la population, et cela, beaucoup plus souvent et plus intensément que les scientifiques occidentaux, les experts en développement et les politiciens ne l'ont supposé à ce jour. La lutte pour le contrôle de la sorcellerie et des pouvoirs occultes est, du point de vue de nombreux Africains, l'un des problèmes fondamentaux du pouvoir politique dans des pays aussi divers que le Bénin, la Côte d'Ivoire, le Nigeria, le Cameroun, le Zaïre (RD Congo) ou même dans le nord de l'Afrique du Sud (voir Fisiy / Geschiere 1990: 148 pour le Cameroun). La preuve effective de ce contrôle de l'occulte ne garantit pas seulement la légitimité personnelle des dirigeants politiques ou des autorités traditionnelles. Dans la mesure où les lois et la jurisprudence des États peuvent efficacement contrôler la propagation de la sorcellerie aux yeux des citoyens, elles favorisent également la légitimité des actes administratifs, et donc le processus de construction de l'État lui-même.

\section{Gagner en légitimité par l'adaptation socioculturelle à l'occulte}

\subsection{Des limites de la recherche de légitimité par l'adaptation socioculturelle du système} juridique

Les puissances coloniales européennes avaient qualifié la magie et la sorcellerie de «philosophie primitive » et de «pratiques arriérées » dans leurs colonies africaines pendant plus d'un demi-siècle. Les accusations de sorcière ont été effectivement bannies, du moins dans la jurisprudence de l'État, dans la mesure où la législation coloniale a fait de l'accusation et de la pratique de la sorcellerie une infraction pénale (voir Nadel 1954: 163-64; Kohnert 1983: 18-19; Ralushai et al 1996: 57). Les maîtres coloniaux ont appliqué cette interdiction non seulement pour des raisons idéologiques, mais aussi pour des intérêts politiques tangibles: ils craignaient des centres de pouvoir concurrents soutenus par des mouvements antisorcellerie, l'instabilité politique et des pertes financières pour le trésor colonial (voir l'exemple de la suppression de le culte anti-sorcellerie dans le nord du Nigéria; Nadel 1954: 196-97; Kohnert 1983: 38, 40).

Dans les régions où les accusations de sorcellerie étaient particulièrement répandues, comme à Nupeland, dans le nord du Nigéria, les Britanniques interdisaient également les cultes secrets et les épreuves, interdisant ainsi aux sorcières locales «blanches » ou sage femmes de combattre les méchantes sorcières aux yeux des intéressés. Le citoyen Nupe se sentait maintenant complètement exposé aux machinations des sorcières et craignait leur propagation sans entraves. Le mépris ostentatoire pour les peurs profondément ressenties de la population en raison de la règle juridique de l'État colonial fondée sur la législation européenne (par exemple le Code Napoléon en Afrique francophone) a contribué de manière significative à son manque de légitimité. Elle a conduit à une profonde méfiance de la population envers les institutions étatiques en général et à l'évasion de la jurisprudence étatique des «blancs » en particulier (voir Nadel 1954: 163-4; Raynal, 1994: 300).

Après l'indépendance - de plus en plus depuis le début des années 1970 - les politiciens et les avocats africains ont tenté de surmonter cet obstacle à la légitimité avec un succès croissant. Dans de nombreux pays d'Afrique sub-saharienne, il y a eu une ré-institutionalisation plus ou 
moins ouverte du crime de sorcellerie dans les lois locales «traditionnelles » ainsi que dans le droit central, tant que les tribunaux traditionnels (coutumiers) n'avaient pas encore poursuivi leur pratique des procès en sorcellerie, comme dans le nord du Nigéria ou en Afrique du Sud, (voir Ralushai et al 1996: 51). En utilisant l'exemple du Cameroun, Fisiy et Geschiere (1990; 1991) ont élucidé ce processus. Des études de cas supplémentaires du Zimbabwe (Chavunduka 1980) et du Zaïre (Duvieusart 1992) sont disponibles.

Dans les pays où le dualisme juridique, dérivé de la domination coloniale, entre la jurisprudence locale «coutumière » et le pouvoir judiciaire de l'État (central), était au moins pratiquement maintenu, cet ajustement devait tenir compte du «bon sens populaire». Des besoins des dirigeants en instrumentaliser des croyances occultes était néanmoins très probablement présente, et y était pratiquée dans la mesure du possible. Dans le nord du Nigéria, où aujourd'hui encore, $80 \%$ de toutes les affaires judiciaires sont tranchées par des tribunaux locaux qui ne sont liés à l'État que formellement, mais en réalité, continuent d'être attachés au système islamique semi-féodal (généralement appelé tribunaux alcali (equivalent au cadi) ou court local), ou également en Afrique du Sud, il existe de fortes indications de dualisme juridique concernant les accusations de sorcellerie (voir Kohnert 1983: 19-25:; Odinkalu 1992; Ralushai et al 1996: 51-57; voir aussi l'études de cas par Raynal 1994 pour la République Démocratique du Congo (ZAR)).

Rouveroy van Nieuwaal (1990: 143-47) rapporte de la région de Mango au nord du Togo que jusqu'à la fin des années 1960, pratiquement aucun tribunal d'État ne traitait des accusations de sorcellerie, tandis qu'au moins $9 \%$ des jugements des autorités locales (autorité coutumière) l'a fait. Contrairement aux juges obligés par la loi coloniale de l'Etat, les chefs traditionnels n'hésitent pas à reconnaître l'existence de la sorcellerie. Ils se vantaient même d'avoir eux-mêmes des pouvoirs occultes qui leur permettaient de découvrir des sorcières. Les sorcières reconnues coupables n'ont été tuées que dans des cas extrêmes. La plupart du temps, ils devaient payer une pénalité ou étaient obligés de faire du travail forcé, par ex. dans les domaines du juge et du chef traditionnel jusqu'à ce qu'ils aient expié leur culpabilité et aient été considérés comme guéris. Dans une étude de cas d'un chef supérieur de N'zara (dans la région des savanes au nord du Togo, 1969-71) enquêtée par Nieuwaal, au moins dix femmes condamnées comme sorcières travaillaient comme travailleuses forcées dans les champs du chef. En 1978, le Togo a officiellement aboli le dualisme juridique entre le droit colonial et le droit commun comme l'un des derniers pays africains. Cependant, la question de savoir si cette abolition a effectivement pris effet ou s'il ne s'agissait que d'une fiction maintenue pour des raisons politiques, comme dans le nord du Nigéria, en Afrique du Sud ou dans la ZAR, est sujette à caution. Au moins la sorcellerie était reconnue par l'État togolais implicitement comme un crime deux ans plus tard (1980) au cours d'une modification du droit pénal (Nieuwaal 1990: 152). Cette reconnaissance était probablement liée à la campagne «d'authenticité » propagée à l'époque, notamment par les régimes despotiques africains, comme le Mobutu du Zaïre ou le régime Eyadéma du Togo, afin de renforcer leur propre légitimité (voir Raynal 1994: 306) et de permettre une instrumentalisation politique des chasses aux sorcières, à discuter plus loin au chapitre 5 .

Il n'est donc pas surprenant que dans un État comme le Nigéria, un juriste de la prestigieuse université Ahmadu Bello, Zaria (Nigeria du Nord), puisse exiger publiquement dans des publications que les lois et les tribunaux nigérians révisent leur attitude négative à l'égard des accusations de sorcellerie (voir Chukkol 1981: 30-36). Il est logique que, dans la même perspective, les juges ivoiriens recommandent sérieusement l'initiation des juges à l'art de la sorcellerie en 1993 afin de pouvoir mieux reconnaître la sorcellerie de l'intérieur (Kohnert 1996: 1352). 
Mais même parmi certains experts juridiques et conseillers occidentaux des gouvernements africains, la demande d'un abandon des normes juridiques eurocentristes vers la reconnaissance de la droit coutumier africaine, y compris le droit de légitime défense contre les attaques de sorcières, semble trouver une oreille attentive. Ainsi, Rouveroy van Nieuwaal (1990: 157), se référant à Renteln (1987), a indiqué que, par exemple, sanctionner la sorcellerie fondée sur le droit de légitime défense était légale. Bien que les juges formés à la perception juridique occidentale se sentiraient mal à l'aise avec un tel point de vue, cela pourrait avoir des conséquences sociales et politiques encore plus graves (par exemple, l'érosion de la légitimité par le gouvernement au pouvoir ou l'instabilité politique) si l'on voulait forcer le changement social par le biais de lois imposées qui serait diamétralement opposé aux visions du monde traditionnelles des sociétés concernées.

Le gouvernement sud-africain actuel semble également supposer une justification analogue. Il veut apparemment lutter contre l'engouement pour les sorcières dans la province du nord (les anciennes homelands Venda, Lebowa et Gazankulu) avec une nouvelle loi adaptée à la culture locale pour supprimer la sorcellerie. Cela était justifié par le fait que cette chasse aux sorcières dure depuis des années et des centaines de femmes sont victimes de l'accusation chaque année (voir également Evans 1992; Minnaar et al 1991; Niehaus 1993; 1995; et chapitre 4). Le nouveau projet de loi, dont le contenu avait également été soutenu par des représentants de l'Église (par exemple l'Église Sioniste et le Zion Christian Church, ZCC, qui est influente dans le nord), devrait en même temps empêcher les émeutes violentes contre de prétendues sorcières et tenir compte des sentiments ressentis de la population et respecter ses convictions.

Le projet de loi du gouvernement sud-africain stipule que « la croyance en la sorcellerie et les pratiques connexes font partie de la culture des Africains en Afrique du Sud » (Ralushai et al 1996: 59) ${ }^{6}$. Le gouvernement a été largement guidé par l'expérience du Zimbabwe voisin, qui a été spécialement étudié à cet effet et dont les réglementations juridiques prétendument éprouvées pour le contrôle de la sorcellerie et des sorciers ou guérisseurs traditionnels ont été largement adoptées (voir Ralushai et al 1996: 64). Cela était justifié entre autres, grâce à l'intégration systématique de plus de 200000 guérisseurs de la province du Nord dans des associations professionnelles et un code d'honneur pour cette profession. Les tendances poursuivies ici pour «domestiquer» la violence personnelle (voir aussi Fisy / Geschiere 1994) et la tendance à monopoliser la violence aux mains de l'État sont indubitables. Ils semblent être explicables et justifiables à la fois du point de vue humanitaire et civiquethéorique (voir Elias 1976).

Cependant, les intentions politiques et les avis juridiques sur lesquels cette demande de réinstitutionnalisation des «vieilles coutumes éprouvées » manœuvrent en terrain contaminé: d'une part, un tel raisonnement ne peut que trop facilement retomber dans le relativisme culturel et juridique, ce qui nie la validité universelle des droits humains fondamentaux,

\footnotetext{
${ }^{6}$ Voir note en bas de page 3. - La Commission d'enquête sur la sorcellerie susmentionnée, qui a élaboré ce projet de loi, a estimé: «Le gouvernement provincial ... a attrapé le taureau par ses cornes à un stade où il s'était déjà déchaîné en raison de la négligence du gouvernement précédent en gestion de la violence de sorcellerie et des meurtres rituels. Trop longtemps, les croyances de sorcellerie et les tueries rituelles avaient été interprétées à tort comme une 'pratique des peuples primitifs' qui, par le biais du mécanisme juridique eurocentriste, serait finalement abandonnée. Cette approche s'est avérée être un désastre. » (Ralushai et al 1996: i ; traduction française, D. K.). - Des représentants d'une commission d'enquête spécifiquement créée par le Conseil sioniste sud-africain des églises (ZCC) sur le problème de la chasse aux sorcières dans la province du Nord ont également exprimé leur opinion au gouvernement en octobre 1995: «... que la restauration de l'ancien ordre africain pourrait peut-être aider à minimiser la violence liée à la sorcellerie. » (voir Ralushai et al 1996: 59 ; traduction française, D. K.).
} 
comme le revendiquent les Nations Unies et la Charte africaine des droits de l'homme et des peuples de l'OUA (Banjul 1981). Cela devient visible dans le domaine juridique, par exemple dans la doctrine de la « défense culturelle », c'est-à-dire le droit à la défense sur la base de son propre patrimoine culturel. Son introduction sous une forme légèrement modifiée a également été appuyée par Rouveroy van Nieuwaal, en utilisant l'exemple du régime autocratique d'Eyadema au Togo (voir Nieuwaal 1990: 155-57). Cela ouvre la porte à des abus de la part du souverain, qui essaie de vendre ses intérêts particuliers comme un intérêt commun basé sur «l'opinion populaire» et la «tradition» (inventée) (voir chapitre 5). Un autre exemple négatif, mieux connu, d'adaptation socioculturelle ou politique des normes juridiques est l'utilisation abusive de la Charte de Banjul, déjà énoncée dans sa conception. Pratiquement tous les droits fondamentaux universels qui y sont mentionnés (par exemple l'article 6-11) sont dévalués par une référence excessive à des lois nationales d'application restrictives et la porte arrière de la «raison d'État» (voir Welch 1992; Wonyu 1992: 35-36). Ainsi, les garanties des droits de l'homme de la Charte sont perforées comme un morceau de fromage suisse. Les régimes autocratiques, comme la dictature militaire nigériane, ne sont que trop heureux de s'appuyer sur la «common law » ou les « objectifs primordiaux de l'État » afin de restreindre massivement les droits humains fondamentaux en appliquant des lois et des décrets.

Certes, les lois et les sanctions ne sont pas le meilleur moyen d'orienter le changement social dans la direction souhaitée. D'autre part, l'adaptation du système juridique au cadre socioculturel d'un pays ne devrait pas conduire à légitimer l'oppression, l'exploitation et un régime d'injustice (voir Raynal, 1994: 307). Des lois et des ordonnances adaptées à la culture locale, combinées à un système juridique adapté en conséquence, peuvent être plus facilement exploitées et instrumentalisées en changeant les alliances politiques ou les intérêts politiques particuliers. Ceci est vrai même si elles correspondent à la perception juridique de la population, que les normes juridiques euro-centrées précédemment en vigueur, par exemple parce que l'enracinement social des premiers est encore moins compréhensible intersubjectivement.

Par conséquent, «l'adaptation socioculturelle » dans le domaine de la législation et du droit, que ce soit pour établir une légitimité, pour garantir la paix intérieure ou comme mesure de développement pour promouvoir l'état de droit, ne devrait être utilisée que de manière très limitée et avec une extrême prudence. Des objectifs bien intentionnés, en particulier dans le domaine de l'occulte, tels que le projet de loi sud-africain cité ci-dessus, pourraient aboutir exactement à l'opposé des objectifs initialement prévus s'ils étaient appliqués dans le cadre de l'État de commandement [Kommandostaat] africain. Les conséquences d'un relativisme juridique exagéré socioculturellement «adapté » ont été révélées par l'exemple du Togo, où comme le montrera le chapitre quatre - la reconnaissance de la sorcellerie dans le système juridique togolais stabilise efficacement la dictature injustice de l'Eyadéma clan.

Cela dit, même tenter de canaliser la croyance en sorcellerie par l'État pourrait, dans certaines circonstances, favoriser encore plus l'engouement des sorcières plutôt que de la freiner. Pourtant, cet effet pourrait même être salué par les systèmes autocratiques d'un point de vue machiavélique, en particulier lorsque l'État peut gagner en légitimité en luttant efficacement contre le fléau croissant des sorcières. Le développement post-colonial au Cameroun peut servir d'exemple. Ici, «l'alliance contre nature » entre l'État et les soi-disant witch-doctors, ou féticheurs (ou Chaman) en français, a consolidé et élargi l'engouement de la sorcellerie. Les accusations de sorcellerie ont été interprétées et combattues comme une subversion dangereuse du pouvoir d'État en général et des efforts de développement d'un État centralisé 
bureaucratique en particulier. Ainsi, l'État a reconnu le féticheur comme le principal témoin de l'acte d'accusation (voir Fisiy / Geschiere 1990: 147-54; Geschiere 1995: 264).

Dans certains pays, comme au Nigéria (Nupeland) ou au Zaïre, le féticheur exploite son entreprise régulièrement et ouvertement - y compris en faisant de la publicité avec des « diplômes » officiels et muni des pouvoirs officiels de l'administration provinciale (voir Kohnert 1983; Duvieusart 1992: 468 / 69). Le féticheur devient ainsi une «profession », semblable à celle d'un médecin ambulatoire, d'un psychothérapeute ou d'un pharmacien de village. La demande vient des chefs de village locaux (voir Kohnert 1983: 23-24 pour le nord du Nigéria; Drucker-Brown, 1993: 539 pour le nord du Ghana), qui sont parfois même contraints sous la menace de la punition de l'État de faire «nettoyer » leurs villages par les sorcières (voir Duvieusart 1992: 468), ainsi que par des chefs de famille ou des individus qui recherchent généralement un coupable pour la catastrophe qui est arrivée à des proches parents. Les chasses aux sorcières et les punitions sont également de plus en plus commercialisées, de la même manière que le trafic des indulgences au Moyen Âge européen (voir Elwert-Kretschmer, 1993; Kohnert 1983: 23-24; voir la thèse "venality" d'Elwert, Elwert 1987).

La propagation de la folie de la sorcellerie ne sera pas nécessairement stoppée par le processus de démocratisation dans le cadre du second souffle de démocratisation en Afrique sub-saharienne au début des années 1990. Au contraire, au Benin et le Cameroun, par exemple, l'élite politique a découvert involontairement une nouvelle source d'accusations de sorcellerie avec le processus de renouveau démocratique: alors que dans le système monopartite centralisé du Cameroun, l'État avait interdit, ou au moins persécuté, les organisations non-gouvernementales locales jusqu'en 1990 , les élites politiques rivales ont maintenant motivé leurs partisans à se battre pour les votes et la loyauté politique dans le pays également. Surtout lorsque les militants du parti ont réussi et que les villageois les ont choisis comme leurs représentants légitimes, les nouveaux dirigeants politiques locaux, les "évolués", se sont inévitablement soumis au schéma de sanctions applicable dans leur village d'origine. Par conséquent, l'augmentation spectaculaire du prestige et de la richesse est invariablement sujette à des soupçons de sorcellerie, surtout si elle n'est pas partagée avec les membres de la communauté sur la base du principe de solidarité mutuelle de la communauté villageoise (voir Geschiere 1996: 85.87-91). En ce sens, "antisocial", dénonce non seulement ceux qui gardent ou consomment pour eux-mêmes leur richesse nouvellement acquise, mais aussi l'agriculteur ou commerçant de village qui accumule et investit son capital individuellement, ou le fonctionnaire, qui «indépendamment de la personne concernée », fournit des services selon des critères de performance apparemment objectifs. Il viole ainsi de manière flagrante les règles traditionnelles de la clientèle locale et les relations de patronage, qui exigent une considération prioritaire des membres de la communauté villageoise ou de la famille élargie.

Encore une autre façon, mais aussi involontaire, la lutte politique de l'ANC contre le régime d'apartheid sud-africain à la fin des années 1980 et au début des années 1990 a conduit à une chasse aux sorcières d'une ampleur sans précédent. Isak Niehaus (1993: 518-27) montre dans des études de cas détaillées que de jeunes militants de l'ANC - similaires aux guérilleros de la lutte de libération du Zimbabwe auparavant (voir Lan 1985: 167,170) - ont utilisé les accusations de sorcellerie et la persécution comme la clé de la légitimité politique, en se profilant dans les villages « conquis » en tant que pouvoirs protecteurs engagés et efficaces et pionniers contre le pouvoir apparemment omniprésent de la sorcellerie. Malheureusement, les combattants contre le régime d'apartheid ont complètement mal compris les instructions politiques de l'ANC, qui a appelé à l'éradication des «pratiques arriérées ». Même, après que les dirigeants de l'ANC ont appelé à la fin de la persécution des sorcières, de nombreux 
militants - comme la population rurale - étaient fermement convaincus que leur chasse aux sorcières favoriserait le processus de modernisation et de démocratisation, qui était apparemment bloqué par les pratiques arriérées des sorcières (voir Evans, 1992: 56; Minnaar et al.1991: 56). Cette chasse aux sorcières avait une dimension politique, à savoir l'approbation de la légitimité des jeunes militants de l'ANC, mais - comme Niehaus (1993; 1995) l'a souligné à juste titre - ce n'était pas une « chasse aux sorcières politique » au sens le plus étroit. En cela, ces accusations de sorcellerie diffèrent fondamentalement des allégations également politiquement motivées et instrumentalisées de meurtre rituel, liés au grigri « $\underline{\text { muti }}$ » dans la même région, qui visaient spécifiquement à combattre l'adversaire politique (voir Minaar et al 1991: 53-55). L'exemple sud-africain fait référence au fait que la construction de la légitimité par l'État et la reconnaissance politique de la croyance a la sorcellerie ont plusieurs dimensions qui ne peuvent être limitées au domaine structurel et fonctionnel. Les accusations de sorcellerie ne sont pas seulement un moyen de poursuivre un objectif politique. La dimension intérieure et existentielle de faire face à la peur et à la découverte de soi des individus et des groupes politiques par le biais d'accusations de sorcières est politiquement au moins aussi importante que leur instrumentalisation consciente. Niehaus (1993) explique cela plus en détail en utilisant l'exemple des militants de l'ANC.

\subsection{Aux limites de la recherche de la légitimité de l'État en soutenant les religions occultes: l'exemple de l'Église kimbanguiste et du vodun}

La dimension existentielle de l'occulte - présentée ci-dessus - affecte la légitimité de l'État non seulement directement et immédiatement sous la forme de la croyance en la magie et la sorcellerie, mais aussi indirectement par l'incorporation d'éléments occultes dans les religions africaines parrainées par l'État. Un exemple relativement bien documenté est l'église kimbanguiste du Zaïre, l'une des plus anciennes et des plus importantes des plus de 6000 soidisant «églises indépendantes africaines ». L'Église de Jésus-Christ sur la terre par le Prophète Simon Kimbangu (EJCSK) est née non seulement du rejet de la complicité des Églises chrétiennes européennes avec le régime inhumain de l'État colonial (voir Körner 1995: 237), mais aussi et surtout en réaction à l'incompréhension des missionnaires et des églises chrétiennes concernant la nécessité pour les croyants d'être protégés des puissances maléfiques surnaturelles. L'élément constitutif du kimbanguisme était la reconnaissance de l'existence de la magie et de la sorcellerie nuisibles, qui ont établi son attrait relativement important et sa suite par rapport aux églises chrétiennes occidentales ${ }^{7}$.

L'église EYCSK a initialement joué un rôle démocratique et émancipateur de promotion du développement. Aux yeux de l'Etat colonial, il était donc plutôt méfiant et, comme la plupart des mouvements religieux autochtones de l'époque, il était immédiatement sujet à la suspicion de subversion politique. En revanche, le lien étroit entre le Kimbanguisme et l'État a entraîné une augmentation urgente de la légitimité du jeune État-nation zaïrois. L'unité des chefs d'État et d'église a démontré aux yeux de la population le contrôle effectif du pouvoir politique dans le domaine du profane comme dans le domaine de l'occulte.

\footnotetext{
7 "L'originalité du kimbanguisme réside dans sa reconnaissance de l'existence des kindoki (les esprits du mal, d. $V$.) invoqués par les ndoki (sorcier, d.V.), dans son recours au pouvoir (supérieur aux simbi) du Saint-Esprit descendu en Simon Kimbangu grâce aux dons transmis au chef spirituel et aux sacrificateurs (condisciples du Prophète) ... C'est ainsi que l'E.J.C.S.K. combat la sorcellerie ou le fétichisme, sans nier son existence, à la différence des églises missionnaires occidentales." (Asch, 1984:219) - Il en va de même pour l'église africaine des Chrétiens Célestes, particulièrement répandue en Afrique de l'Ouest, et les Rose-Croix.
} 
Cependant, l'ambivalence politique d'une telle légitimation de la domination magique et religieuse de l'État est rapidement devenue évidente: après que la direction des kimbanguistes a été complètement prise en charge par l'État à partir de 1965, l'église kimbanguiste a dégénéré en une sorte de "changement constantinien » vers un pilier important de l'appareil d'État répressif de Mobutu (voir Körner 1995: 238; Asch 1983). Avec le deuxième processus de démocratisation du Zaïre, qui a commencé au début des années 1990, et la mort du chef du EJCSK, Josèphe Diangienda, en juillet 1992, le Kimbanguisme a eu l'occasion historique de contribuer à l'établissement d'un nouvel État constitutionnel sur le sol zaïrois en opposition à le régime affaibli de Mobutu. On espérait qu'elle pourrait raviver ses traditions démocratiques de base du début de l'ère coloniale, semblable à ce que l'Église catholique locale faisait depuis 1990 (voir Körner 1995: 237). Pourtant, jusqu'à présent, le Kimbanguisme a apparemment laissé passer cette opportunité.

Les pratiques religieuses - quelle que soit leur orientation - ont toujours une dimension éminemment politique dans les pays africains comme dans de nombreux autres pays du tiersmonde (voir Bayart 1993: 15, 19-162). Les efforts récents visant à moderniser les églises et les religions africaines et à les reconnaître officiellement comme un élément de soutien de l'État s'accompagnent souvent de justifications supplémentaires. Par exemple, ils pourraient constituer un point de départ pour ancrer une éthique africaine authentique qui serait bénéfique pour l'établissement d'une société civile organisée démocratiquement. Cela s'applique particulièrement aux religions avec de forts éléments occultes comme les églises indépendantes africaines en général et le vodun ouest-africain en particulier. Ce dernier a été combattu non seulement par des missionnaires chrétiens et des maîtres coloniaux, mais aussi par des dirigeants africains comme Mathieu Kérékou, dictateur militaire du Bénin socialiste (1972-1989), berceau du vodun, en tant que « relique de la féodalité ${ }^{8}$.

Cependant, les efforts pour réhabiliter le vodun, afin de promouvoir le développement démocratique, s'efforcent surtout de changer le contenu et l'éthique de ce système de croyances, une évolution qui s'éloigne des limites d'un culte secret vers un culte ouvert à tous. $\mathrm{Au}$ moins divers nouveaux cultes vodun au Bénin (par exemple, alafia, atingali) ne sont pas liés à des régions, des groupes ethniques ou des associations familiales spécifiques (voir Kadya Tall 1995: 235). Au Bénin, ces tentatives sélectives ont été encouragées par la

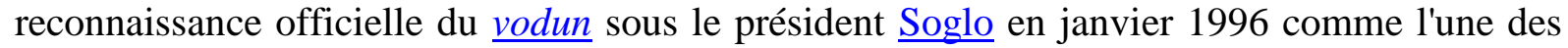
principales religions populaires du pays aux côtés du christianisme et de l'islam.

Les partisans du développement de mouvements religieux et d'une politique africain « épurés » «d'en bas » ont pu se référer à des modèles de développement dans d'autres pays, présentés lors d'une conférence internationale sur le vodun à Ouidah (Bénin) au printemps 1993, parrainée par Soglo ( pour la littérature pertinente, voir Gbégnonvi 1993: 3.4).

En Haïti, par exemple, où le vodun avait été importé avec des esclaves africains, la religion est devenue un refuge et une alternative pour les défavorisés en temps de crise, contrairement au projet de développement social échoué de l'élite ${ }^{9}$. La «révolution qui naît de l'esprit du

\footnotetext{
${ }^{8}$ Voir Kadya Tall 1995: 196-99; Gbégnonvi 1993: 6. - Les nouveaux gouvernements des États africains ont pour la plupart repris l'aversion des dirigeants coloniaux contre les églises africaines autochtones, du moins lorsque ces dernières ont également remis en question le pouvoir du nouveau gouvernement africain. Un autre exemple en est la suppression par l'État guinéen (Bissau) de Nino Vieira du mouvement religieux de base njangnjang - fondé par des femmes balantes à la fin des années 1980 (voir Kohnert 1996: 1350).

${ }^{9}$ «Le vodun est la forme de religion la plus décentralisée ... un système de croyance qui ,pousse par le bas . Plutôt que d'être imposé par le haut, comme la plupart des religions, il est, élevé et nourri dans la famille, dans
} 
vodun » a joué un rôle décisif dans le processus de démocratisation après la chute du régime Duvalier en 1986. Cette révolution avait été soutenue par la structure sociale du vodun (voir Rotberg 1976) et sa dimension socio-culturelle, y compris des groupes de beat populaires à orientation politique avec leurs chansons séditieuses (par exemple Boukman Eksperyans). Pour la première fois dans l'histoire d'Haïti, le vodun a été publiquement reconnu comme

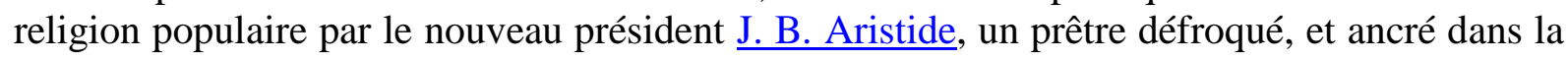
constitution (Nicolas 1994: 111, 115).

Cependant, l'exemple d'Haïti montre en même temps l'ambivalence de la fonction politique du vodun, qui, comme d'autres religions et systèmes de croyances occultes, peut être utilisée pour légitimer toute forme de pouvoir. Pourtant, en général, il a été utilisé comme instrument d'oppression ou d'exploitation ou comme « opium du peuple » selon l'aphorisme marxiste (voir Kohnert 1996: 1349). Après sa prise de pouvoir en 1957, le président haïtien François Duvalier a pris le vodun comme un moyen efficace et redouté de terroriser la population. Ce faisant, il s'est assuré du soutien actif de la majorité des prêtres vaudous, qu'il a instrumentalisés et a même fait de certains d'entre eux les chefs locaux de son groupe de voyous notoires, les tonton macoutes (Nicolas 1994: 115; Rotberg 1976: 362 -365).

$\mathrm{Au}$ Bénin également, les commandements traditionnels du vodun ont principalement servi à renforcer les structures familiales traditionnelles et ethniques sociales dont ils sont issus (voir Gbégnonvi 1993). Théoriquement, la tentative d'établir le vodun en tant que religion populaire soutenue par l'État pourrait se traduire par l'enseignement de valeurs éthiques fondamentales universelles (plutôt conservatrices) (par exemple la solidarité, l'honnêteté, le courage, le respect et la loyauté) et par la protection institutionnelle souhaitée, également pour l'expansion de la société civile béninoise et la sécurisation durable de la légitimité de l'État.

En cas de conflit entre les valeurs locales traditionnelles de la communauté des croyants et les systèmes de valeurs nationaux de rang supérieur (par exemple la lutte contre la corruption et le népotisme), le vodun encourage et légitime (dans des conditions par ailleurs identiques) la maintien des systèmes traditionnels de gouvernement, le favoritisme et les réseaux de clientèle avec toutes les conséquences négatives qui en résultent (voir Elwert-Kretschmer 1995). En tant que religion basée sur la peur, la vengeance et l'occultisme, le vodun dans son ensemble est probablement plus un obstacle à la démocratisation au niveau national. Cela a été confirmé entre autres par le professeur Béninois Roger Gbégnonvi qui rejette avec véhémence les récentes tentatives de réhabilitation du vodun ${ }^{10}$.

\footnotetext{
les villes, et dans les champs. C'est de la common loi '... Ce sont des adorateurs démocratiques ordinaires ayant un accès direct. » (Rotberg 1976:345; traduction en français, D.K.)

${ }^{10}$ «Le vodun est force de terreur avant d'être force de mobilisation, et c'est pourquoi après l'avoir servi et s'être servi de lui, on peut encore se détacher de lui comme on s'éloigne d'un volcan dont on sait qu'il peut se réveiller à tout moment pour ravager sans rémission les terres qu'il a pourtant fertilisées. ... Quand le peuple, toutes tendances confondues, tient pour suspect le vodun, nous ne pouvons pas continuer à soutenir contre lui la théorie du vodun, ferment de liberté et d'épanouissement. Le vodun ne peut pas remplir cette fonction parce qu'il n'est pas transparent. ... parce qu'une telle prétention est aux antipodes de sa nature ... le vodun (est) ...inapte à toute réhabilitation dans sa forme d'hier parce qu'hier il a pactisé avec le crime (l'esclavage, $d$.V.). Il ne peut pas réhabiliter dans sa forme d'aujourd'hui parce qu'aujourd'hui, il refuse de reconnaître qu'il a pactisé hier avec le crime. ... Le vodun est disqualifié parce qu'il n'aime pas l'homme. »(Gbégnonvi 1993:7-8).
} 


\section{Sur l'instrumentalisation des systèmes de croyances occultes pour soutenir la pouvoir illégitime}

Ceux qui veulent utiliser des systèmes de croyances occultes pour promouvoir la légitimité de l'État se lancent dans un acte d'équilibrage risqué comme indiqué ci-dessus. Il est trop facile, pour ceux qui veulent échanger des gains de légitimité bon marché basés sur les particularités socioculturelles en Afrique, de courir le risque de sombrer dans l'illégitimité.

L'utilisation de la magie et de la sorcellerie pour soutenir l'ordre illégitime, c'est-à-dire pour supprimer et exploiter les personnes socialement défavorisées, peut se produire de diverses manières. Un exemple est l'utilisation offensive de la croyance en la magie et la sorcellerie pour combattre activement l'adversaire politique: cela se produit souvent simultanément à plusieurs niveaux. Les méthodes vont de simples menaces, plus ou moins voilées ${ }^{11}$, la guerre psychologique, la psycho-terreur, jusqu'à l'emprisonnement dans des camps de concentration pour de prétendues «sorcières » au Ghana, Togo et autres pays de l'Afrique occidentale (Kohnert, 1996), et enfin le meurtre d'opposants, comme par «magie noire » (par exemple par empoisonnement). En règle générale, dans les conditions de «1 'État de commandement africain » (voir Bierschenk, Elwert et al. 1993) et du système de patronage en vigueur dans la plupart des pays africains, ces sanctions menacent non seulement les opposants politiques directs, mais aussi les membres de leur famille et leurs partisans, ce qui rend déjà la simple menace extrêmement efficace.

Des autocrates africains tels que Bokassa (République centrafricaine), Mobutu (Zaire, République démocratique du Congo) et Eyadéma (Togo) semblent avoir utilisé ces instruments particulièrement virtuoses et sans scrupules (voir Schatzberg 1993: 448-450; Nguza, 1982: 29-30, 63; Toulabor 1986: 124-131). La légitimation de ces régimes par l'occulte était généralement basée sur des campagnes de propagande bien planifiées pour renforcer le charisme personnel des autocrates. Bien que la revendication de légitimité soit ainsi liée à certains dirigeants qui étaient décrits comme uniques, elle ne se limitait pas à cette personne mais était inextricablement liée au clan et au régime de ces dirigeants, à savoir l'appareil d'État qui garantissait effectivement leur domination, analogue à l'état fasciste du régime hitlérien.

Même si le culte de la personnalité était généralement orchestré à l'avance et mis à jour périodiquement, il serait faux de supposer que l'instrumentalisation de l'occulte se faisait de manière purement machiavélique sans sa propre préoccupation intérieure. Les potentats comme Eyadéma sont eux-mêmes irrémédiablement empêtrés dans la logique de la magie et de la sorcellerie et agissent en conséquence en élargissant leur culte de légitimité de la personnalité, comme le «Eyadémaïsme » (voir Toulabor 1986: 124-131, 133-229; Ellis 1993). Dans ce qui suit, ceci sera examiné plus en détail à l'aide de l'exemple des chasses aux sorcières sous le régime de Gnassingbé Eyadema.

Le témoignage public lors de la Conférence nationale souveraine togolaise en août 1991 a offert une occasion unique de jeter un bref coup d'œil derrière le rideau d'obscurité qui entourait l'occulte politique de «purification et punition ». Des témoignages de délégués de la conférence devant des caméras de télévision en direct, appuyés de données et de chiffres concrets, ont donné un premier aperçu de toute l'étendue du règne occulte d'Eyadéma, qui ne

\footnotetext{
${ }^{11}$ par exemple, des menaces d'émirs dans le nord du Nigéria pendant la campagne électorale du gouvernement local de 1986 avec une allusion à leurs pouvoirs magiques supposés, selon la devise: « Je peux voir qui choisit mes candidats et mes volontés et agir en conséquence », voir Kohnert 1983.
} 
s'est pas arrêté à son village natal de Pya (centre du Togo, près de la ville de Kara) et sa propre famille. Le délégué Toyi Assih, qui, comme le président appartenait à l'ethnie Kabyé, a témoigné, par exemple, de la déportation et du meurtre en noyant et tuant au moins six villageois nommés pour sorcellerie en 1984 et 1985. Onze autres personnes de Pya étaient déportés dans le camp de concentration de Mandouri (nord du Togo) destiné aux soi-disant sorcières, où ils ont également été tués ${ }^{12}$.

Assih a souligné que, contrairement à la croyance populaire, les Kabyés n'étaient nullement épargnés des mesures terroristes du régime. Certaines exécutions arbitraires ont même eu lieu en présence du chef de l'État en personne. L'un des cas ci-dessus concernait le chef traditionnel et père adoptif d'Eyadéma, un autre a été assassiné parce qu'il aurait causé la mort $\mathrm{du}$ père biologique d'Eyadéma par la sorcellerie. Parmi les personnes déportées pour sorcellerie se trouvait une femme qui avait donné naissance à Eyadéma, etc. (voir Ellis 1993: 472).

Les auditions de témoins au cours de la Conférence nationale ont mis en évidence deux choses: premièrement, la démonstration du pouvoir, des rituels et des symboles occultes ont non seulement servi de portée extérieure, comme moyen d'assurer la légitimité et la domination despotique, mais ils ont également été utilisée à des fins privées. Deuxièmement, l'utilisation de l'occulte pour des meurtres rituels pour garantir le régime autocratique n'était pas limitée à Eyadéma lui-même. Des représentants du régime Eyadéma étaient impliqués à tous les niveaux de l'administration publique, du ministre de l'Intérieur au simple chef de canton (voir Ellis 1993: 472). Ainsi, les accusations de sorcellerie n'ont pas servi seules, ni même principalement, à garantir le pouvoir et l'autorité personnels, mais à assurer la domination d'un groupe stratégique ou d'une faction de l'élite politique du pays par tous les moyens (occultes et laïques) que le système rendait disponibles à l'appareil d'État adapté à leurs besoins ${ }^{13}$.

Le simple témoignage d'un chef de canton était souvent suffisant pour arrêter les prétendues sorcières et les garder secrètement en tant que travailleurs forcés dans une ferme sous la surveillance des chefs traditionnels ou dans des camps de concentration (voir Dovi 1992: 14). À cet égard, le «Dossier Mandouri » présenté à l'attention internationale à la Conférence nationale en août 1991 par Robert Ahlonko Dovi, président de la Commission nationale togolaise des droits de l'homme $(\mathrm{CNDH})$, est révélateur. La visite, qui a eu lieu pour la première fois en novembre 1990 à la demande de la ligue togolaise des droits de l'homme et des organisations internationales des droits de l'homme, dans un «camp de concentration de sorcières » à Mandouri (nord du Togo, à environ $85 \mathrm{~km}$ à l'est de Dapaong à la frontière de Bénin). Au moment de l'enquête, 41 personnes étaient toujours accusées d'être des sorcières ou d'avoir commis de la sorcellerie. Ils y ont été détenus indéfiniment sans inculpation officielle. La plupart d'entre eux (appartenant pour la plupart à l'ethnie Kabyè) ont été expulsés par les chefs traditionnels responsables de leurs régions d'origine respectives. Le plus grand nombre (32) de détenus des camps de concentration du Mandouri provenait de la

\footnotetext{
${ }^{12}$ Voir le quotidien français Fraternité-Matin (Abidjan), « Togo - Conférence nationale », 9. août 1991, p. 24.

13 Les moyens de gouvernement «laïques » concernaient en particulier les militaires, une sorte de garde prétorienne, que le régime Eyadéma avait développés en une des plus grandes armées du monde par habitant au cours de ses près de trente ans de règne. Cependant, cela n'a été possible que grâce à l'aide active au «développement» des anciennes puissances coloniales, la France et l’Allemagne, pendant des décennies. Les différents services de sécurité de l'État étaient attachés à Eyadéma par des liens ethniques et familiaux. Le Rassemblement du peuple togolais (RPT) avec ses sous-organisations (voir aussi Toulabor 1986), qui, après cinq ans de démocratisation, a échoué en raison de la stratégie terroriste de Eyadéma, a retrouvé son ancien pouvoir étant donné la division interne de l'opposition et la manipulation des élections en 1996.
} 
préfecture de Kozah (dont 15 de Pya, lieu de naissance d'Eyadéma). De manière significative, l'existence de camps de concentration togolais est connue depuis longtemps, du moins selon les rumeurs, même au-delà des frontières du Togo ${ }^{14}$.

Dans le contexte de la fracture mondiale Est-Ouest, il n'était tout simplement pas envisagé de rompre la «paix du cimetière » au Togo, qui jusqu'en 1989 avait été surnommée «la Suisse de l'Afrique » par de nombreux politiciens dans les milieux politiques bienveillants envers le régime Eyadéma. Jusqu'en 1989, le gouvernement fédéral allemand en général, et son aide au développement en particulier, avaient apparemment d'autres normes pour évaluer la légitimité du régime Eyadéma que quelques années plus tard ${ }^{15}$. À cet égard, le Togo est un bon exemple du fait que la survie de régimes illégitimes soutenus par des prétentions occultes au pouvoir, en Afrique aussi, n'est souvent possible qu'avec un soutien supplémentaire et une attribution externe de « légitimité » par des gouvernements étrangers.

\section{Conclusions: la démocratie en Afrique ne peut pas être induite par la sorcellerie}

À une époque où le succès de l'aide au développement en Afrique est de plus en plus remis en cause, de nouveaux concepts et solutions sont nécessaires. Même les donateurs à vocation technocratique antérieurs, comme la Banque mondiale, soulignent aujourd'hui l'importance du développement du capital humain et, en outre, ils soulignent l'importance de la dimension sociale et politique (par exemple la bonne gouvernance, la responsabilité, la transparence) de l'ajustement structurel. Lier les innovations aux conditions socioculturelles et aux ressources endogènes est aujourd'hui, non sans raison valable, une condition préalable à la réussite des programmes de développement ${ }^{16}$. Cette tendance, à prendre davantage en compte la dimension socioculturelle dans la coopération au développement, correspond à un retour aux systèmes de valeurs locaux du partenaire. En raison des nombreuses tentatives infructueuses de démocratisation des systèmes politiques africains depuis l'indépendance, il est également logique «d'endogénéiser » l'aide à la démocratisation de l'Afrique (voir Diaby-Pentzlin 1996). Trop souvent, l'adaptation inappropriée des modèles de démocratie occidentale formelle n'a pas abouti à une participation plus efficace de la population, mais seulement à un échange d'élites politiques.

\footnotetext{
14 Déjà, le 27 juin 1985, il y avait une interpellation du Parti social-démocrate d'Allemagne (SPD) (Dr. Diederich, Berlin) au Bundestag, demandant s'il était vrai qu'avec «l 'aide à l'équipement» allemande (par exemple, des mission militaires de pionnier et équipement de police) une route d'accès par un « camp de travail pour des criminels présumés » avait été construit près de Kazaboua (préfecture de Sotouboua). Le ministre d'État de l'époque, Jürgen Möllemann (ministère des Affaires étrangères) du Parti démocratique libre (FDP), a rejeté avec véhémence cette accusation en faisant référence à des prétendues particularités socio-culturelles africaines, où il n'y a pas le même « sentiment de souveraineté », et en plus «il y a des opinions différentes sur certains concepts tels que l'éducation et la morale que les nôtres ». Il a ajouté que selon les informations togolaises, le camp en construction n'était qu'un «établissement d'enseignement pour les jeunes difficiles à éduquer ». En fait, apparemment «seulement» des voies d'accès au KZ-Kazaboua ont été construites sous la direction des pionniers allemands travaillant comme conseillers et formateurs au Togo (Bundestag allemand, procès-verbal de la 10e législature, 149 session, Bonn, 27 juillet, 1985, p. 11122 -25). - A l'initiative de la CNDH, le camp de concentration de Mandouri a été dissous au printemps 1991: «Ainsi, depuis cette date, il n'existe plus de 'sorciers' à Mandouri en espérant qu'il n'existera plus jamais de 'sorcier », dit-il dans le dossier Mandouri (Dovi 1992: 15). Il reste à voir si cet espoir se révèle viable, compte tenu de la reprise quasi-totale des anciens postes de pouvoir sous le régime d'Eyadéma jusqu'en 1996, notamment grâce au soutien actif de la France.

${ }^{15}$ En raison des violations massives des droits de l'homme du régime Eyadéma, la coopération allemande au développement avec le Togo a été officiellement suspendue en grande partie à partir de février 1993.

${ }^{16}$ Le concept-cadre en matière socioculturel du BMZ, qui a été adopté en 1992 comme base de la coopération au développement de l'État allemand, en est un exemple remarquable.
} 
Il existe cependant des limites étroites à l'adaptation des systèmes de décision politique aux conditions socioculturelles locales dans les pays africains. Cela s'applique en particulier aux tentatives de résoudre l'une des questions fondamentales de l'aide au développement, la question de la légitimité des partenaires de l'État dans la coopération au développement. Selon la tradition, aux yeux de la plupart des Africains, le pouvoir profane et occulte sont inextricablement liés. Contrairement à une opinion commune, l'occulte - comme indiqué cidessus - sert non seulement à soutenir le pouvoir personnel charismatique ou traditionnelle, mais aussi le pouvoir juridique de l'État. Cependant, si l'adhésion politique et le sentiment juridique de la population sont si étroitement liés à des systèmes de croyances occultes, l'aide démocratique fondée sur des conditions socioculturelles devient un dangereux équilibre: il est donc constamment nécessaire de vérifier, si la promotion durable de la participation, et le processus d'émancipation des défavorisés, en reliant ce processus à leurs systèmes de valeurs et de croyances, ne glissent pas dans le relativisme culturel contre-productif, qui soutient consciemment ou inconsciemment - les régimes d'injustices répressives.

En principe, comme de nombreux autres systèmes de croyances (par exemple la légitimation des croisades et des deux guerres mondiales par le christianisme), la croyance en la magie et la sorcellerie peuvent servir à légitimer n'importe quelle pouvoir, à la fois despotique et légale démocratique. Comme d'autres systèmes de croyances, les croyances africaines sur la magie et la sorcellerie ne peuvent pas être vérifiées de manière intersubjective selon des critères scientifiques. Elles dépassent donc largement la vérification académique. Le risque d'abus est particulièrement important dans le cas de systèmes de croyances occultes, entre autres en raison du «secret » excessif typique qui les entoure, des restrictions d'accès (organisation dans des sociétés secrètes; souvent discriminatoires à l'égard des femmes) et de la hiérarchisation des connaissances (selon différents niveaux d'initiation, etc.), ce qui rend plus difficile le partage de savoir. Cela est plutôt préjudiciable au développement d'une société démocratique et autodéterminée. D'un autre côté, une divulgation et une démocratisation renforcées des processus décisionnels à tous les niveaux (du gouvernement jusqu'à l'assemblée du village) seraient une condition préalable nécessaire (mais probablement pas suffisante) pour freiner la croyance en la magie et la sorcellerie. Plus de transparence et une société plus ouverte, fondée sur la concurrence égale des idées et des systèmes politiques, sont les meilleurs garants d'une démocratisation durable. 


\section{Bibliographie}

Ardener, Edwin (1970): Witchcraft, economics and the continuity of belief, in: Douglas, Mary (ed.), "Witchcraft confessions and accusations, In: Witchcraft Confessions and Accusations, edited by Mary Douglas. Tavistock Publications, London, pp. 141-60

Asch, Susan (1984): L'église du prophète Kimbangu - De ses origines à son rôle actuel au Zaïre. Paris : Le Monde diplomatique, Janvier 1984, p. 18

Awolalu, J. Omosade (1979): Yoruba beliefs and sacrificial rites. London

Bayart, Jean-François (eds.)(1993): Religion et modernité politique en Afrique noire - Dieu pour tous et chacun pour soi. Paris: Karthala

Bierschenk, Thomas / Elwert, Georg / Kohnert, Dirk (1993): The long-term effects of development aid: empirical studies in rural West Africa. Economics (Tübingen), vol. 47, No. 1, pp. 83-111

Chavunduka, G. L. (1980): Witchcraft and the law in Zimbabwe. Zambwezia (Harare), vol. 8, No. 2, pp. $129-147$

Chukkol, Kharisu Sufiyan (1981): Supernatural beliefs and the criminal law in Nigeria - A critical appraisal. Zaria (Nigeria), Ahmadu Bello University

Comaroff, Jean / Comaroff, John (eds.) (1993): Modernity and its malcontents: Ritual and power in postcolonial Africa. University of Chicago Press

Diaby-Pentzlin, Friederike (1996): Rechtsprojekte auf dem Weg zur Endogenisierung. Entwicklung und Zusammenarbeit $(\mathrm{E}+\mathrm{Z})$, Vol. 37, No. 11, pp. 310-11

Douglas, Mary (eds.) (1970): Witchcraft accusations and confessions. London : Routledge

Dovi, Ahlonko (1992): Dossier Mandouri. in: Les Echos, Magazine d'information de la Commission Nationale des Droits de l'Homme (CNDH), No. Special, pp. 14-17

Drucker-Brown, Susan (1993): Mamprusi witchcraft, subversion and changing gender relations. Africa (London), vol. 64, No. 4, pp. 531-549

Duvieusart, Léopold s.j.(1992): Cas de recherche de sorcellerie en milieu rural au Zaïre. Suggestions sur la conduite à tenir par les autorités de l'Etat. Zaïre-Afrique (Kinshasa), pp. 457-471

Elias, Norbert (1976): Über den Prozeß der Zivilisation - Soziogenetische und psychogenetische Untersuchungen. vol. 2, Wandlungen der Gesellschaft: Entwurf zu einer Theorie der Zivilisation. Frankfurt/M.: Suhrkamp

Ellis, Stephen (1993): Rumour and power in Togo. Africa (London), vol. 63, No.4, pp. $462-476$

Elwert, Georg (1987): Ausdehnung der Käuflichkeit und Einbettung der Wirtschaft. In: Klaus Heinemann (eds.): Soziologie wirtschaftlichen Handelns. Sonderheft Nr. 28 der Kölner Zeitschrift für Soziologie und Sozialpsychologie, pp. 300-321

Elwert-Kretschmer, Karola (1995): Vodun et contrôle social au village. Politique Africaine, vol. 59, pp. 102-119

Engel, Ulf / Hofmeier, Rolf / Kohnert, Dirk / Mehler, Andreas (eds.)(1995): Wahlbeobachtung in Afrika. Arbeiten aus dem Institut für Afrika-Kunde, No. 90, Hamburg

Evans, Jeremy (1991): Muti murders - Ritual responses to stress. Indicator SA, vol. 8, No. 4, pp. 46-48 
Evans, Jeremy (1992): On brule bien les sorcières - Les meurtres muti et leur repression. Politique Africaine, vol. 48, pp. 47-57

Evans-Pritchard, E.E. (1937): Witchcraft, Oracles and Magic among the Azande. Oxford

Fisiy, C. / Geschiere, P. (1990): Judges and witches, or how is the state to deal with witchcraft? Cahiers d'Etudes Africaines, vol. 118, pp. 135-156

Fisiy, Cyprian / Geschiere, Peter (1991): Sorcery, witchcraft and accumulation: regional variations in south and west Cameroon. Critique of Anthropology, vol. 11, No. 3, pp. 251-278

Fisiy, Cyprian F. (1990): Le monopol juridictionnel de l'Etat et le règlement des affaires de sorcellerie au Cameroun. Politique africaine, vol. 40, pp. 60-71

Gbégnonvi, Roger (1993): Le vodun disqualifié. mimeo, Cotonou

Geschiere, Peter (1988): Sorcellerie et modes populaires d'action politique chez les Maka (Cameroun). In: Bogumil Jewsjewicki (eds.): Savoire, pratiques populaires en Afrique. Paris :

Geschiere, Peter (1995): Sorcellerie et politique en Afrique: La viande des autres. Paris : Karthala

Geschiere, Peter (1996): Sorcellerie et politique: les pièges du rapport élite-village. Politique Africaine, vol. 63, pp. 82-96

Geschiere, Peter / Fisiy, Cyprian (1994): Domesticating personal violence: witchcraft, courts and confessions in Cameroon. Africa, vol. 64, No. 3, pp. 323-341

Geschiere, Peter / Konings, Peter (eds.) (1993): Itinéraires d'accumulation au Cameroun. Paris: Karthala

Gluckman, Max (1944): The Logic of African Science and Witchcraft. Human Problems in British Central Africa, No. I (1944), pp. 61-71. Reprint in: Marwick (eds.), 1975, pp. 321-331

Kadya Tall, Emmanuelle (1995): De la démocratie et des cultes voduns au Bénin. Cahiers d'Etudes Africaines, vol. 36, No. 1, pp. 195-208

Körner, Peter (1995): Die Transformation der kimbanguistischen Kirche im Prozeß des Wandels der Gesellschaftsverfassung in Zaïre. In: Pitschas, Rainer / Sülzer, Rolf (eds.): Neuer Institutionalismus in der Entwicklungspolitik. Berlin, pp. 235-53

Kohnert, Dirk (1983): Indicators of social and political conflict in African societies: on the articulation of witchcraft among the Nupe, Northern Nigeria. Working Paper No. 32, Forschungsschwerpunkt Entwicklungssoziologie, Universität Bielefeld

Kohnert, Dirk (1988): Socialism without liberation - Land reclamation projects in Guinea-Bissau. in: Sociologia Ruralis, No. 2/3, pp. 161-175

Kohnert, Dirk (1996): Magic and witchcraft: implications for democratization and poverty-alleviating aid in Africa. World Development, vol. 24, No. 8, pp. 1347-1355

Macfarlane, A.D.J. (1970): Witchcraft and conflict. In: Macfarlane, A.D.J.: Witchcraft in Tudor and Stuart England. London, Chap. 15. reprinted in: Marwick, Max (eds.)(1975), Witchcraft and Sorcery. Selected Readings. Harmondsworth, pp. 296-304

Macfarlane, A.D.J. (1970a): Definitions of witchcraft. In: Macfarlane, A.D.J.: Witchcraft in Tudor and Stuart England. London, App. 2; reprinted in: Marwick, Max (ed.)(1975): Witchcraft and Sorcery. Selected Readings. Harmondsworth, pp. 41-44 
Marwick, Max (1964): Witchcraft as a social strain-gauge. Australian Journal of Sociologie, vol. 26, PP. 263-68; reprint in: Marwick, Max (eds.)(1975): Witchcraft and Sorcery. Selected Readings. Harmondsworth, pp. 280-295

Marwick, Max (eds.) (1975): Witchcraft and Sorcery. Selected Readings. Harmondsworth

Mayrargue, Cédric (1995): Le religieux et les législatives de mars 1995 au Bénin. Politique Africaine, vol. 58, pp. 158-162

Minnaar, Anthony / Offringa, Dirkie / Payze, Catharine (1991): The witches of Venda: Politics in magic potions. Indicator $S A$, vol 9, No 1, pp. 53-56

Multhaupt, Tamara (1990): Hexerei und Antihexerei in Afrika. München: Trickster Wissenschaft

Nadel, Siegfried F. (1952): Witchcraft in four African Societies. The American Anthropologist, vol. 54, pp. 18-29; reprinted in: Marwick, Max (eds.)(1975): Witchcraft and Sorcery. Selected Readings. Harmondsworth, pp. 264-279

Nadel, Siegfried F. (1954): Nupe Religion. London; Routledge, reprint 1970

Nguza, Karl I Bond (1982): Mobutu - ou l'incarnation du mal Zaïrois. London: Bellew Publishing Co Ltd.

Nicolas, Alrich (1994): Die kulturpolitischen Wurzeln Haitis. Vaudou als Institution für ein neues Gesellschaftsprojekt. In: Rieger, Gerhard (eds.): Die Karibik zwischen Souveränität und Abhängigkeit. In: Blätter des IZ3W, Freiburg, Januar 1994, pp. 111-116

Niehaus, Isak A., (1993): Witch-hunting and political legitimacy: Continuity and change in Green Valley, Lebowa, 1930-91. Africa, vol. 63, No. 4, pp. 498-530

Niehaus, Isak A., (1995): Witches of the Transvaal Lowveld and their familiars: Conceptions of Duality, power and desire. Cahiers d'Etudes africaines, vol. 138-139, vo. 35-2-3, pp. 513-540

Nieuwaal, E.A.B. van Rouveroy van (1990): Sorcellerie et justice coutumière dans une societé togolaise. Journal of Legal Pluralism and Unofficial Law, vol. 29, pp. 137-162

Nogueira, Amadeu (1947): O 'Irâ' na Circumscriçâo de S. Domingos. Bolletim Cultural da Guiné Portuguesa. (Bissau/Lisbon), No. 7, pp. 711-716

Odinkalu, Anselm Chidi (1992): Justice denied - The Area Court system in the Northern States of Nigeria. Civil Liberties Organisation, Ibadan

Ralushai, N.V. / Masingi, M.G. / Madiba, D.M.M. / Van den Heever, J.A. / Mathiba, T.J. / Mphaphuli, M.E. / Mokwena, M.W. / Vele Ndou, P.N. / Matabane, D. (1996): Report of the commission of inquiry into witchcraft violence and ritual murders in the Northern Province of the Republic of South Africa. Report submitted to "His excellency the honourable member of the executive council for savety and security, Northern Province, Advocate Seth Nthai". No place, no publisher given, 274 p., plus Annex

Raynal, Maryse (1994): Justice traditonelle, justice moderne - Le devin, le juge et le sorcier. Paris : Harmattan

Reining, Conrad C. (1966): The Zande Scheme: an anthropological case study of economic development in Africa. Evanston, Illinois: Northwestern University Press, 255 p.

Renteln, A.D. (1987): Culture and culpability: a study of contrast. Beverly Hills Bar Association Journal, Winter 1987/88, pp. 17-27 
Rotberg, Robert I. (1976): Vodun and the politics of Haiti. In: Kilson, M. / Rotberg, R. (eds.): The African Diaspora. Harvard Univ. Press, pp. 342-365

Schatzberg, Michael G. (1993): Power, legitimacy and 'Democratization' in Africa. Africa, vol. 63, No. 4, pp. 445-461

Sulikowski, Ulrike (1993): 'Sie geben eine Party für die Götter und die Götter kommen'. Überlegungen zu Vodun, Besessenheitskulten und dem Diskurs über Afrika. In: Fillitz, Thomas et al. (eds.). Kultur, Identität und Macht. Frankfurt/M.

Toulabor, Comi M. (1986): Le Togo sous Eyadéma. Paris: Karthala

Tseayo, J. I. (1975): Conflict and incorporation in Nigeria. Zaria: Gaskiya Corporation

Turner, V.W. (1964): Witchcraft and sorcery: taxonomy versus dynamics. Africa, vo. 34, No. 4, pp. $319-24$

Welch, Claude Jr. (1992): Human rights charter. West Africa, Nov. 16-22, 1992, p. 1952

Wilson, K.B. (1992): Cults of violence and counter-violence in Mozambique. Journal of Southern African Studies, vol. 18, No. 3, pp. 527-582

Wonyu, Emmanuel (1992): Un support juridique pour la démocratie en Afrique: La charte Africaine des Droits de l'homme et des Peuples. Afrique 2000, Febr. 1992, pp. 29-45 
Abstract: [The impact of the occult on state legitimacy and aid for democratization in Africa]

Among politicians and development experts in Africa alike there is a growing awareness of the ever decreasing importance of the belief in magic and witchcraft on political decision-making since pre-colonial times. Demonstrating control over occult forces as a means of strengthening their legitimacy had been the prerogative of traditional rulers and their marabouts for over a century. Today, it is effective for the modern political elite and the modern state as well. A growing number of African states have officially recognized the existence of witchcraft and magic, and they are adapting the colonial law imposed accordingly. In addition to magicoreligious belief systems, represented by vodun or independent African churches (for example, the Kimbanguists), benefiting from controlling witchcraft, are promoted by African political leaders to strengthen the legitimacy of both the class state policy and governance. At the same time, development experts have tried to take into account the cultural dimension of socio-cultural development. They called for an "endogenization" of development aid. This call was justified, because "endogenization" should be seen as a prerequisite for sustainable aid. However, under certain conditions it can be ambiguous and dangerous too. Regarding the consideration of occult belief it can lead to the promotion of the illegitimate state and the violation of basic human rights.

Zusammenfassung: [Der Einfluss des Okkulten auf staatliche Legitimität und Demokratisierungshilfe in Afrika]

Unter Politikern und Entwicklungsexperten in Afrika wächst das Bewusstsein für die immer geringer werdende Bedeutung des Glaubens an Magie und Hexerei für die politische Entscheidungsfindung seit vorkolonialer Zeit. Die Demonstration der Kontrolle über okkulte Kräfte als Mittel zur Stärkung ihrer Legitimität war über ein Jahrhundert lang das Vorrecht der traditionellen Herrscher und ihrer Marabouts. Heute ist es auch für die moderne politische Elite und den modernen Staat wirksam. Immer mehr afrikanische Staaten haben die Existenz von Hexerei und Magie offiziell anerkannt und passen das auferlegte Kolonialrecht entsprechend an. Neben magisch-religiösen Glaubenssystemen, vertreten durch Vodun oder unabhängige afrikanische Kirchen (zum Beispiel die Kimbanguisten), die von der Kontrolle der Hexerei profitieren, werden von afrikanischen politischen Führern gefördert, um die Legitimität sowohl der Klassenstaatspolitik als auch der Regierungsführung zu stärken. Gleichzeitig haben Entwicklungsexperten versucht, die kulturelle Dimension der soziokulturellen Entwicklung zu berücksichtigen. Sie forderten eine „Endogenisierung“ der Entwicklungshilfe. Diese Forderung war berechtigt, weil die „Endogenisierung“ als Voraussetzung für nachhaltige Hilfe zu sehen ist. Unter bestimmten Bedingungen kann es jedoch auch mehrdeutig und gefährlich sein. Bei der Berücksichtigung okkulten Glaubens kann dies zur Förderung des illegitimen Staates und zur Verletzung grundlegender Menschenrechte führen. 